\title{
Covid-19: Who is at Risk and How to Risk Stratify for Poor Outcome?
}

\author{
Syed Raza*
}

\author{
Consultant Cardiologist and Physician, Awali Hospital, \\ Bahrain
}

\section{Introduction}

The SARS-COV-2 is not even 90 days old and much is already known about it by scientists and healthcare professionals across the globe. However, there are still areas of knowledge gap and uncertainty. This has left us with many unanswered questions and members of public in fear and confusion.

First, very little is known about the original source of this virus and the other modes of transmission of infection in addition to droplet. Whether it is air borne or has orofecal transmission in addition is still for anybody to guess. It is also not clear as to what proportion of patients of COVID-19 exhibit no symptoms but has the potential to transmit the infection. There are many claims that have been circulating on the internet, social media and print media regarding the treatment and therapeutic options that are available to fight this deadly virus. The fact however is, that there is no fact in these statements. These claims come from studies which are only observational, anecdotal and have paucity of data from a very small study population and very short follow up. There is virtually no scientific study design and therefore will be unacceptable by healthcare scientific community at large. These claims and statements therefore are not only false and misleading but also dangerous.

The real challenge that is now being faced by healthcare professionals is testing and screening people for COVID-19. There is also some limitation in risk stratifying individuals and patients as to who are likely to contract the disease and who would have a worse or better outcome. The answer to these questions have not been simple and straightforward so far. However, based on limited experiences from areas such as in China and Italy we have gained some insight on certain demographics and parameters that would help us to identify and assess these risks. Some of these risk factors are as listed below:

1. Age: This has been recognized as the most important determinant in risk stratifying individuals and patients. People of age more than 65 years are thought to be most vulnerable and have been found to have poorer outcome. Some younger people are also being infected but these individuals have been found to be smokers, have chronic lung disease or have compromised immune system.

2. Sex: Male preponderance has been observed for poor clinical course and outcome.
*Corresponding author

Syed Raza, Consultant Cardiologist and Physician, Awali Hospital, Bahrain

Submitted: 05 May 2020; Accepted: 12 May 2020; Published: 23 July 2020

3. Co-morbidities: One of the main poor indicators that have been identified is obesity. Obese patients particularly in European hospitals are more likely to end up in intensive care unit requiring artificial ventilatory support and other life support measures. Chronic lung disease, chronic kidney disease, advanced liver failure, malignancy, uncontrolled diabetes mellitus and cardiovascular disease including heart failure.

4. Patients on chemotherapy, immune-suppressants or steroid

5. Undernourished

6. Chronic alcoholism

7. Poor personal and food hygiene

8. Cultural norms of greetings i.e. Handshakes, hugging or kissing

9. Some lab parameters - Low serum albumin, low white cell count, high D-Dimer, A blood group - These are however not validated.

It is therefore important that screening for SARS-COV-2 is done with some lateral thinking. When most country's economy is suffering, and healthcare resources are already stretched, judicious use of facilities are of paramount importance. This can in some way be achieved by identifying risk factors in individuals and patients and thereafter risk stratifying them for screening or therapy. This may also involve using risk stratification scoring system and clinical pathways. Above all it is the clinical judgement and decision for individualized care which will surpass any other methods.

\section{References}

1. Content source: National Center for Immunization and Respiratory Diseases (NCIRD), Division of Viral Disease.

2. The Asia Executive Editor of The Lancet, Helena Hui Wang, chats with Bin Cao about clinical course and risk factors for mortality of adult inpatients with COVID-19 in Wuhan, China. Podcast

3. Daniel Kaul, MD reviewing Wu C et al. (2020) Risk Factors for ARDS and Progression to Death Among COVID-19 Patients. JAMA Intern Med.

4. Chaomin $\mathrm{Wu}$, Xiaoyan Chen, Yanping Cai, Jiałan Xia, Xing Zhou, et al. (2020) Risk factors associated with acute respiratory distress syndrome and death in patients with coronavirus disease 2019 pneumonia in Wuhan, China. JAMA Intern Med.

Copyright:@2020 Syed Raza.,This is an open-access article distributed under the terms of the Creative Commons Attribution License, which permits unrestricted use, distribution, and reproduction in any medium, provided the original author and source are credited. 